\title{
Low Incidence of Colonization and No Cases of Disseminated Mycobacterium avium Complex Infection (DMAC) in Brazilian AIDS Patients in the HAART Era
}

Ângela Gadelha, Náurea Accácio, Beatriz Grinzstejn, Valdiléa Veloso, Liane Braga da Silveira, Fátima Fandinho, Maria Helena Saad, Maria Cristina Lourenço and Valeria Rolla

\author{
Evandro Chagas Institute, National Institute \\ of Quality Control, Laboratory of Hanseniasis, \\ Oswaldo Cruz Institute, National School of \\ Public Health, Laboratório of Bacteriology \\ and Hanseniasis, Fiocruz/RJ, Rio de Janeiro, \\ RJ, Brazil
}

\begin{abstract}
Objective. Evaluate the incidence of mycobacterial disease and the colonization of the respiratory and gastrointestinal tracts by Mycobacterium avium complex (MAC) bacteria in AIDS patients. Methods. Inclusion criteria: HIV-positive individuals with at least one $\mathrm{CD}_{4}^{+}$count $<100$ cells $/ \mathrm{mm}^{3}$. Exclusion criteria: Mycobacterial disease and MAC prophylaxis. Stool, sputum, and blood cultures were prospectively obtained every month from September, 1997, to December, 1999. The incidence was calculated using Poisson regression. Survival was estimated by the Kaplan Meier method and the Cox proportional hazard model. Results. We followed-up 79 patients during a median period of 428 days. Blood cultures $(n=742)$ were negative for all mycobacteria. Positive cultures ( 25 samples) were obtained from non-sterile sites: Stools $(19 / 703$ specimens $=2.7 \%)$ and sputum $(14 / 742$ specimens $=1.9 \%)$. MAC was isolated in $7 / 703$ stool samples $(1 \%)$ and $1 / 32$ sputum specimens $(0.1 \%)$. The incidence of patient colonization with $\mathrm{MAC}$ was $0.09 /$ year $(\mathrm{CI}=0.05-0.18)$. $\mathrm{CD}_{4}$ counts in patients colonized with MAC were below $100 \mathrm{cells} / \mathrm{mm}^{3}$ in only 2 out of 8 cases. Restoration of $\mathrm{CD}_{4}^{+}$counts $>100$ cells $/ \mathrm{mm}^{3}(\mathrm{HR}=0.18 ; \mathrm{CI}=0.05-0.70)$ predicted a lower risk of death $(\mathrm{P}<0.05)$ but was not protective for $M A C$ colonization $(H R=0.52 ; C I=0.62-4.35, P=0.55)$. Conclusion. The absence of DMAC infection in colonized individuals argues in favor of a HAART protective effect against; DMAC; however, restoration of $\mathrm{CD}_{4}$ counts did not protect patients against MAC colonization. Key Words: DMAC, colonization, AIDS, mycobacteria.
\end{abstract}

Disseminate Mycobacterium avium complex infection (DMAC) became the most frequent opportunistic disease (OD) of bacterial origin in AIDS patients [1], until the HAART era. This infection became more evident since progress in prophylaxis for OD, and antiretroviral treatment, has allowed AIDS patients to survive longer, even with advanced immunodeficiency and $\mathrm{CD}_{4}{ }^{+} \mathrm{T}$ cell counts below 100 cells $/ \mathrm{mm}^{3}[2,3]$.

Received on 22 April 2002; revised 12 September 2002. Address for correspondence: Dr. Valeria Rolla. Evandro Chagas Institute. Av. Brasil, 4365, Manguinhos, Rio de Janeiro, Zip code: 21045-900.

The PAPES II - FIOCRUZ program supported this work.

The Brazilian Journal of Infectious Diseases 2002;6(5):252-257 (C) 2002 by The Brazilian Journal of Infectious Diseases and Contexto Publishing. All rights reserved.

1413-8670
Rio de Janeiro is an endemic area for tuberculosis (TB) and HIV in Brazil. Some Brazilian studies have detected MAC in bone marrow aspirates [4] and blood cultures [5] of AIDS patients presenting clinical signs and symptoms of mycobacterial disease in the pre-HAART era. To our knowledge the incidence of DMAC infection remains to be determined in Brazil.

\section{Objective}

The aim of this study was to evaluate the colonization of the respiratory and gastrointestinal tracts of HIVinfected patients with atypical mycobacteria and to determine the incidence of mycobacterial disease in immunosuppressed AIDS patients treated with highly active antiretroviral therapy (HAART). 


\section{Material and Methods}

This was a prospective observational study carried out to evaluate the incidence of DMAC infection and other mycobacterial diseases in immunosuppressed AIDS patients. Our study was conducted at the Instituto de Pesquisa Clinica Evandro Chagas, Fiocruz, Rio de Janeiro, a center for research on HIV/AIDS and other infectious diseases. Inclusion criteria: All HIV-positive patients who had at least one $\mathrm{CD}_{4}^{+}$count below 100/ $\mathrm{mm}^{3}$ were considered eligible. Exclusion criteria: Patients under treatment for tuberculosis or DMAC infection at the time of enrollment in the study and the use of MAC prophylaxis (MAC prophylaxis is not formally recommended in Brazil and has not been adopted in our center).

Sputum (spontaneous or induced), stools and blood were obtained monthly from September, 1997, to December, 1999. All samples were cultivated in Lowënstein-Jansen medium. Blood cultures were performed using the lysis-centrifugation "home made" method [6)] that has been used in our center since 1994. Stools and sputum were seeded after decontamination by the Petroff method. Mycobacteria were identified by standard biochemical methods and by PCR. Additional samples were requested from the patients' health care providers whenever indicated clinically. All patients were interviewed to obtain their medical and antiretroviral treatment histories. Viral load and $\mathrm{CD}_{4}^{+}$counts were prospectively evaluated every 4 months. Our protocol was analyzed and approved by The Ethics Committee of FIOCRUZ and all patients gave informed consent to participate in the study.

Statistical analysis. Baseline $\mathrm{CD}_{4}$ counts for all included patients were below 100 cells $/ \mathrm{mm}^{3}$. Patients were separated into two groups during the study, according to $\mathrm{CD}_{4}$ counts: Those who had at least one $\mathrm{CD}_{4}$ count above 100 during follow-up $\left(\mathrm{CD}_{4}>100\right.$ cells $\left./ \mathrm{mm}^{3}\right)$ and those who did not return to counts above $100\left(\mathrm{CD}_{4}\right.$ $<100$ cells $\left./ \mathrm{mm}^{3}\right) . \mathrm{CD}_{4}$ counts associated with colonization were those determined closest to the moment MAC was isolated.
We considered colonization by atypical mycobacteria to be present when no clinical signs or symptoms of mycobacterial disease were observed and at least one culture for atypical mycobacteria from a non-sterile site was positive. Disseminated mycobacterial infection was defined as a positive culture from a normally sterile site.

Survival was estimated by the Kaplan Meier method and the curves were compared by the log rank test. The risks of death and of colonization were determined by the Cox proportional hazard model.

Study endpoints were the development of DMAC infection, death or loss to follow-up. For the risk of colonization with MAC, the endpoint considered was a positive culture for MAC. The cohort was followed up monthly during 27 months.

The rate of incidence of colonization was estimated by a Poisson regression.

The confidence interval stipulated was $95 \%$.

\section{Results}

We followed up 79 patients for a mean period of $733(\mathrm{CI}=683-782)$ days and a median of 428 days. Of these individuals, $57(72 \%)$ were men and $22(28 \%)$ were women. Nine patients died of AIDS, corresponding to an $11 \%$ death rate, $69(89 \%)$ were finished because the study had ended and one $(1.3 \%)$ was left the study because of a car accident.

We made 2,177 cultures for Mycobacteria in 79 patients (Table 1). Among these cultures, 742 were made from blood samples, 732 from sputum and 703 from stool samples. All blood cultures were negative for Mycobacteria. Positive cultures $(n=33)$ were obtained from stools ( 19 of $703=2.7 \%$ ) and sputum ( 14 of $732=1,6 \%)$ in 25 individuals. One bronchoalveolar lavage (BAL) and one lymph node (LN) biopsy were also positive for M. tuberculosis. MAC was isolated from 8 (out of 2177 specimens $=0.3 \%$ ) of 79 asymptomatic patients (10\%). Only one specimen was positive for each patient. The rate of incidence per person per year of mycobacterial colonization (all specimens of mycobacteria excluding tuberculosis cases) was $0.26(\mathrm{CI}=0.18-0.39)$, and the rate of 
colonization with MAC was 0.09 per year $(\mathrm{CI}=0.05-$ $0.18)$. The incidence of tuberculosis was 0.08 per year $(\mathrm{CI}=0.04-0.14)$.

Tuberculosis (TB) was diagnosed in 9/79 patients (11\%) during follow-up, although $M$. tuberculosis was isolated in only $7 / 79$ patients $(9 \%)$. $M$. tuberculosis was isolated from five sputum specimens, one BAL and one LN biopsy. All cultures were negative for two of the cases of TB, however these patients responded to specific treatment.

During our study all patients were treated with HAART except TB patients during rifampicin treatment. Baseline $\mathrm{CD}_{4}$ counts were $\leq 50$ cells $/ \mathrm{mm}^{3}$ in 43 patients and $>50$ cells $/ \mathrm{mm}^{3}$ in 36 patients. In 59 of $79(75 \%)$ of the cases we observed an increase in $\mathrm{CD}_{4}$ counts above $100 / \mathrm{mm}^{3}$ during follow-up. The remaining 20 patients never achieved counts above 100 cells $/ \mathrm{mm}^{3}$. Interestingly, median $\mathrm{CD}_{4}{ }^{+}$counts made close to tuberculosis diagnosis were lower (55 cells $/ \mathrm{mm}^{3}$ ) than those made close to MAC colonization (136 cells $/ \mathrm{mm}^{3}$ ) and only 2 out of 8 patients colonized with $\mathrm{MAC}$ had $\mathrm{CD}_{4}$ counts below 100 cells $/ \mathrm{mm}^{3}$ within 3 months of the colonization date.

Baseline $\mathrm{CD}_{4}^{+} \mathrm{T}$ cell counts $>50 / \mathrm{mm}^{3}(\mathrm{HR}=0.16$; CI 0.02-0.25) and an increase of $\mathrm{CD}_{4}^{+} \mathrm{T}$ cell counts $>100 / \mathrm{mm}^{3}(\mathrm{HR}=0.18$; CI 0.05-0.70) were associated with a lower risk of death $(\mathrm{P}<0.05)$ (Figure 1) but the risk of colonization was similar in both groups: those with $\mathrm{CD}_{4}$ counts $>100$ cells $/ \mathrm{mm}^{3}$ and $<100$ cells $/ \mathrm{mm}^{3}\left(>100\right.$ cells $/ \mathrm{mm}^{3} \mathrm{HR}=0.52 ; \mathrm{CI}$ $=0.62-4.35, \mathrm{P}=0.55)$. Mucosal colonization with MAC (yes, mean survival $=703$ days; $\mathrm{CI}=563-$ 842 and no, mean survival $=732$ days $; \mathrm{CI}=679$ 785 ) and TB diagnosis (yes, mean survival $=677$ days; $\mathrm{CI}=523-831$ and no, mean survival $=733$ days; $\mathrm{CI}=681-786$ ) were not predictors of survival $(\mathrm{P}=1$ and 0.9 respectively), although the small number of cases in each category limited the analysis.

\section{Discussion}

In 1996, the treatment of HIV infection underwent considerable change. Protease inhibitors (PI) and non- nucleoside analogue reverse transcriptase inhibitors (NNRT) became available for clinical practice as part of a combined drug regimen in a highly active antiretroviral treatment (HAART) [7]. Adecrease in morbidity, mortality and incidence of many AIDSassociated OD was reported worldwide [8-13]. Some cases of MAC infection, however, changed their clinical presentation from disseminated to localized forms, such as abscesses and scrofula and other cases were reported in association with antiretroviral resistance [14].

We observed a very low prevalence and rate of incidence of MAC colonization in the respiratory and gastrointestinal systems of patients from Rio de Janeiro. MAC was isolated from only $0.3 \%$ of the cultivated specimens ( $10 \%$ of the patients), and from only one specimen in all of them.

No cases of DMAC were observed over a 23 month follow-up and no cases of DMAC infection have been diagnosed in our Hospital in patients treated with HAART since 1997. In Brazil, before the HAART era, MAC was isolated from feces [15], blood cultures (16\%) from patients presenting unexplained fever who were selected for clinical suspicion of DMAC [5] and from $18.4 \%$ of bone marrow specimens [4]. No other Brazilian study has evaluated the incidence of DMAC infection in immunosuppressed asymptomatic patients. In the United States, Chin et al. reported a very high prevalence of MAC in respiratory and gastrointestinal tracts before the HAART era. The risk of MAC bacteremia at that time was estimated at $\approx 60 \%$ within a year for patients diagnosed with MAC in either the respiratory or gastrointestinal tracts [16].

Recent studies have evaluated the incidence of OD after HAART. Studies performed in Canada, Europe and the U.S. have shown a significant decrease in morbidity and mortality in the HAART era [8-12]. In Brazil, an initial decline in mortality was also observed from 1991 to 1994 but a significant decrease was coincident with the distribution of PI by the Ministry of Health, even in regions with a high incidence such as the Northeast and South [13]. In all of these studies, the incidence ofDMAC infection decreased significantly after the introduction of HAART, although MAC prophylaxis was also recommended to AIDS patients 
Table 1. Mycobacteria isolated in sputum stools and blood cultures of 79 patients from 1997 to 1999 in a median of 428 days in IPEC - Fiocruz

\begin{tabular}{lllll}
\hline Mycobacteria species & Patients & Sputum & Stools & Blood \\
\hline M. tuberculosis & $7(8.9 \%)$ & $8(1.1 \%)$ & $0(0 \%)$ & $0(0 \%)$ \\
M. avium intracellulare & $8(10.1 \%)$ & $1(0.1 \%)$ & $7(1 \%)$ & $0(0 \%)$ \\
M. flavescens & $2(2.5 \%)$ & $0(0 \%)$ & $2(0.3 \%)$ & $0(0 \%)$ \\
M. kansasii & $1(1.3 \%)$ & $1(0.1 \%)$ & $0(0 \%)$ & $0(0 \%)$ \\
M. scrofulaceum & $1(1.3 \%)$ & $3(0.4 \%)$ & $3(0.4)$ & $0(0 \%)$ \\
M. gordonae & $1(1.3 \%)$ & $0(0 \%)$ & $1(0.1 \%)$ & $0(0 \%)$ \\
M. terrae & $1(1.3 \%)$ & $0(0 \%)$ & $1(0.1 \%)$ & $0(0 \%)$ \\
*Mycobacteria notidentified & $3(3.8 \%)$ & $1(0.1 \%)$ & $5(0.7 \%)$ & $0(0 \%)$ \\
Number of positives & $25(31.6 \%)$ & $14(1.9 \%)$ & $19(2.7 \%)$ & $0(0 \%)$ \\
Total evaluated & $79(100 \%)$ & $732(100 \%)$ & $703(100 \%)$ & $742(100 \%)$ \\
\hline
\end{tabular}

* insufficient to all biochemical tests.

Figure 1. Survival analysis considering patients who achieve $\mathrm{CD}_{4}^{+} \mathrm{T}$ cell counts above $100 \mathrm{~mm}^{3}(\mathrm{~N}=59)$ and those who were unable to increase baseline values $(\mathrm{N}=20)$

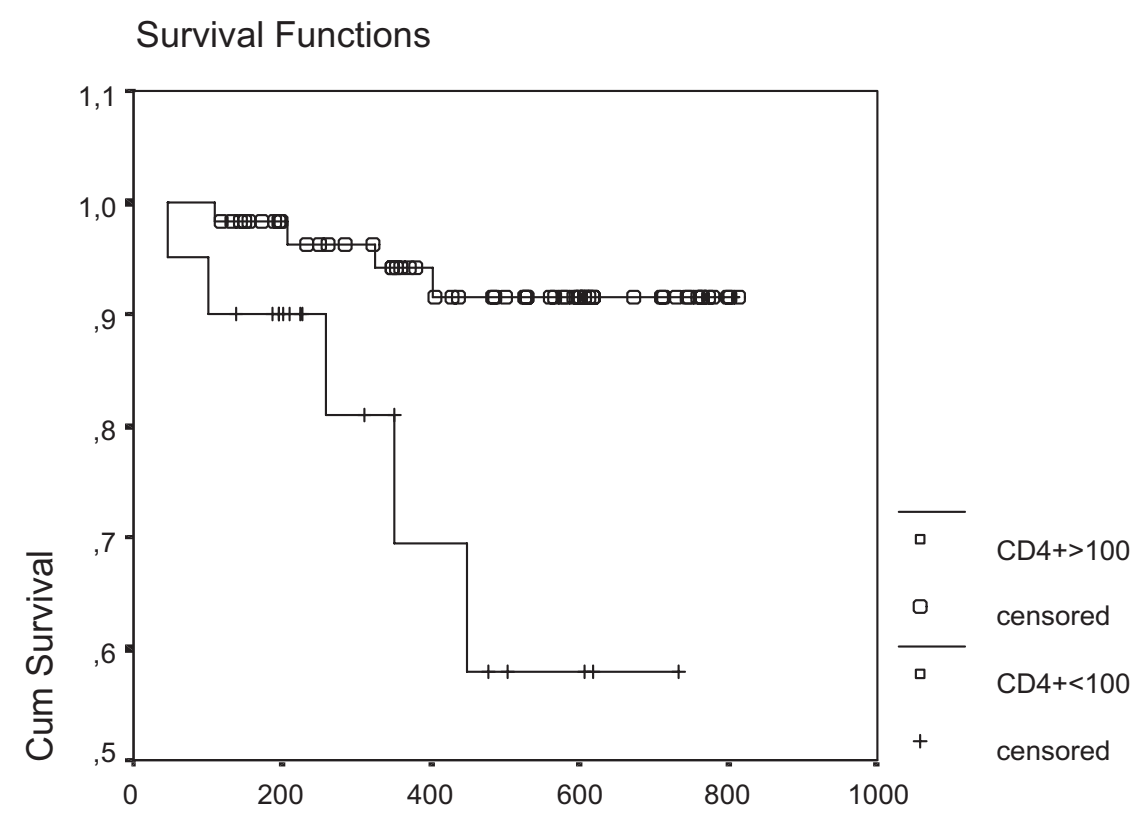

Time in days 
with $\mathrm{CD}_{4}$ cell counts below 100 cells $/ \mathrm{mm}^{3}$ in the U.S. and Europe, which contributed to the observed decline. In those regions, the overall incidence of TB and DMAC was estimated in individuals treated with HAART and a 20-fold reduction was observed for DMAC versus a 5-fold reduction for TB [17] suggesting a higher impact of HAART in the incidence of DMAC infection.

MAC prophylaxis also has had an important role in decreasing the incidence of DMAC, as reported in a large cohort study [18]. Some recent studies however, have shown that prophylaxis against DMAC could be safely withheld in patients with $\mathrm{CD}_{4}$ counts above 100 cells $/ \mathrm{mm}^{3}$ in response to HAART because the risk of DMAC in this population was found to be low [19]. A Brazilian group also reported recovery from DMAC infection without specific treatment in patients treated with HAART [20]. In our study, an increase in baseline $\mathrm{CD}_{4}^{+}$counts after the initiation of antiretroviral therapy could have contributed to the low incidence of DMAC infection observed in our study, although the risk of colonization was similar in patients with counts below and above 100 cells $/ \mathrm{mm}^{3}$ $(\mathrm{HR}=0.97)$. Other factors, such as the prevalence of mycobacteria in the environment may explain why patients with low $\mathrm{CD}_{4}^{+}$counts without MAC prophylaxis were not heavily colonized. Nevertheless no cases of DMAC were observed in patients under HAART in our center.

Brazilian Guidelines do not formally recommend MAC prophylaxis in our country and no data about DMAC incidence have been reported to encourage this recommendation.

\section{Aknowledgements}

We thank the Day-Hospital team for their special dedication: Luci Alves, Solange Alves da Cruz, Sonilde Mello Fazollo and Marcia Regina Lyra da Silva.

\section{References}

1. Nightingale S.D., Byrd L.T., Southern P.M., et al. Incidence of Mycobacterium avium-intracellulare complex bacteremia in human immunodeficiency viruspositive patients. J Infect Dis 1992;165:1082-5.
2. Chaisson R.E., Gallant J.E., Keruly J.C., Moore R.D. Impact of opportunistic disease on survival in patients with HIV infection. AIDS 1998; 12:29-33.

3. Rolla V.C., Gadelha A.J., Accacio, et al. Opportunistic diseases incidence and survival in AIDS patients who experienced very low $\mathrm{CD}_{4}^{+}$cell counts In The Protease Inhibitors Era. IX International Conference on Aids, Durban, South Africa 2000 (TuPe 3341).

4. Barreto J.A., Palaci M., Ferrazoli L., et al. Isolation of Mycobacterium avium complex from bone marrow aspirates of AIDS patients in Brazil. J Infect Dis 1993;168:777-9.

5. Grinsztejn B., Fandinho F.C., Veloso V.G., et al. Mycobacteremia in patients with the acquired immunodeficiency syndrome. Arch Intern Med 1997; 157:2359-63.

6. Fandinho F.C., Grinsztejn B., Veloso V.G., et al. Diagnosis of disseminated mycobacterial infection: testing a simple and inexpensive method for use in developing countries. Bull World Health Organ 1997;75(4):361-6.

7. Carpenter J.C.C., Fischl A.M., Hammer M.S. Antiretroviral Therapy for HIV Infection in 1996. Recommendations of the International AIDS Society - USA Panel. JAMA 1996;276:78-86.

8. Chaisson R.E., Gallant J.E., Keruly J.C., Moore R.D. Impact of opportunistic disease on survival in patients with HIV infection. Aids 1998; 12:29-33.

9. Hogg M.A., Heart K., Yip B., et al. Improved survival among HIV-infected individuals following initiation of antiretroviral therapy. JAMA 1998;279:450-4.

10. Palella F.J., Delaney K.M., Moorman A.C., et al. Declining morbidity and mortality in patients with advanced human deficiency virus infection. N Engl J Med 1998;338:(13)853-60.

11. Brodt H.R., Kamps B.S., Gute P., et al. Changing incidence of AIDS-defining illnesses in the era of antiretroviral combination therapy. AIDS 1997;11:1731-8.

12. Ledergerber B., Egger M., Telenti A. AIDS-Related opportunistic illness and potent antiretroviral therapy. JAMA 2000282(23):2220-6.

13. Fonseca M.G., Barreira D. A evolução da mortalidade por aids no País, segundo sua distribuição geográfica. Boletim Epidemiológico - AIDS ANO XIII N ${ }^{\circ} 3-36^{\mathrm{a}}$ a 52ª SEMANAS EPIDEMIOLÓGICAS, Out. a Dez. 2000.

14. Jacobson M.A., French M. Altered natural history of AIDS-related opportunistic infections in the era of potent combination antiretroviral therapy. AIDS 1998; $12:$ S157-63.

15. Grinsztejn B., Werneck E.B., Lourenço M.C., et al. Isolation of Mycobacteria from the faeces of AIDS patients. IX International Conference on AIDS, Berlin, 1993. 
16. Chin D.P., Hopewell P.C., Yajko D.M., et al. Mycobacterium avium complex in the respiratory or gastrointestinal tract and the risk of $M$. avium complex bacteremia in patients with human immunodeficiency virus infection. J Infect Dis 1994; 169:289-95.

17. Kirk O., Gatell J.M., Mocroft A., et al. Infections with Mycobacterium tuberculosis and Mycobacterium avium among HIV infected patients after the introduction of highly active antiretroviral therapy. Am J Resp Crit Care Med 2000;162:865-72.

18. Benson C., Williams P.L., Cohn D.L., et al. Clarithromicin or Rifabutin alone or in combination for primary prophylaxis of Mycobacterium avium complex disease in patients with AIDS: a randomized double-blind placebo controlled trial. J Inf Dis 2000;181:1289-97.

19. El-Sadr W.M., Burman W.J., Grant L.B., et al. Discontinuation of prophylaxis against Mycobacterium avium complex disease in HIV-infected patients who have a response to antiretroviral therapy. $\mathrm{N}$ Engl J Med 2000;343(5):1085-92.

20. Hadad D., Lewi D., Pignatari A., et al. Resolution of Mycobacterium avium complex bacteremia following highly active antiretroviral therapy. Clin Infect Dis 1998;26:758-9. 